Himat Vaghadia MB BS FFARCS MHSC FRCPC, * Carolyn Fowler MB CHB FANZCA

\title{
Can nurses screen all outpatients? Performance of a nurse based model
}

Purpose: This paper outlines and evaluates a nurse based model for screening outpatients that is utilized in our free standing Surgical Day Care Centre (SDCC).

Methods: For 668 outpatients presenting at our SDCC, the attending anesthesiologist completed a study survey that was designed to identify: completeness of history; important concerns as judged by the pre-admission nurse; whether the patient was seen in the anesthesia preadmission clinic (PAC) for a consultation; if there was a delay in SDCC, the duration and reasons for the delay; whether in the opinion of the attending anesthesiologist the patient should have had an anesthetic consultation; whether the patient was canceled and the reason for cancellation.

Results: A nurse based model for screening all outpatients in a university affliated tertiary hospital day care unit had an accuracy of $81 \%$, specificity of $86 \%$, sensitivity of $46 \%$ and a negative predictive value of $92 \%$. The cancellation rate with this model was $I .4 \%(8 / 551)$ and the case delay rate was $3.4 \%(19 / 551)$. The referral rate to anesthesiology staff was $17.5 \%(117 / 668)$ and the referral rate to the PAC for anesthetic consultation was $5.1 \%(34 / 668)$.

Conclusions: The use of the nurse based model allowed for the efficient use of anesthesia and surgical day care centre resources. The model was better at 'ruling out' patients who do not need to be seen by anesthesiology ahead of the day of surgery rather than 'ruling in' patients who need to be seen by anesthesiology.

Objectif : Le présent article trace les grandes lignes d'un modèle-nursing, pour la sélection des patients ambulatoires, utilisé à notre Centre de chirurgie d'un jour (CC)).

Méthode : L'anesthésiologiste en service a rempli un questionnaire d'étude, pour les 668 patients qui se sont présentés à notre CC], conçu pour enregistrer : l'anamnèse complète, les préoccupations importantes selon l'infirmière de la préadmission; le fait d'une consultation à la clinique de préadmission en anesthésie (CPA); la survenue d'une annulation et sa raison d'être; l'opinion de l'anesthésiologiste quant à la pertinence d'une consultation anesthésique préalable; le fait d'une annulation de l'opération et la raison qui l'a motivée.

Résultats : Un modèle-nursing pour la sélection des patients ambulatoires, dans une unité de soins ambulatoires d'un hôpital tertiaire afflié à l'université, présentait une fiabilité de $81 \%$, une spécificité de $86 \%$, une sensibilité de $46 \%$ et une valeur de prédiction négative de $92 \%$. Avec ce modèle, le taux d'annulation a été de 1,4\% $(8 / 55 \mathrm{I})$ et le taux de report des interventions a été de 3,4\% (19/55I). On a dirigé $17,5 \%(117 / 668)$ des patients vers le personnel d'anesthésiologie et $5,1 \%$ (4/668) vers la CPA pour une consultation anesthésique.

Conclusion : L'utilisation d'un modèle basé sur la pratique du nursing a permis l'usage efficace des ressources du centre de chirurgie et d'anesthésie ambulatoires. Le modèle a été meilleur pour «exclure" des patients qui n'avaient pas besoin de rencontrer l'anesthésiologiste avant le jour de l'opération plutôt que pour «accepter» ceux qui en avaient besoin.

From the Departments of Anaesthesia, Vancouver General Hospital and Health Care \& Epidemiology, ${ }^{*}$ University of British Columbia, Vancouver, BC.

Address correspondence to: Dr Himat Vaghadia, Department of Anaesthesia (LSP 2449), Vancouver General Hospital, 855 West 12 th

Avenue, Vancouver, B.C. V5Z 1M9 Canada. Phone: 604-875-4304; Fax: 604-875-5209; E-mail: hvaghadi@vanhosp.bc.ca Accepted for publication August 22, 1999 
A $S$ outpatient surgery continues to change, a variety of approaches have been used for screening patients. ${ }^{1}$ Recently, Badner et al. described a screening tool for anesthesia preadmission assessment that was found to be effective and allowed for more efficient use of medical personnel. ${ }^{2}$ The Surgical Day Care Centre (SDCC) of Vancouver General Hospital has an annual case load of approximately 10,000 patients and has relied exclusively on physician based preoperative screening for patient preassessment. Since many patients are healthy and do not require medical intervention before surgery, a physician assessment may be unnecessary and may strain physician resources. Recently, our institution developed a nursebased screening model, that utilises a screening tool similar to that described by Badner. ${ }^{2}$ The purpose of this report is to describe our nurse based model and to evaluate its effectiveness.

\section{Methods}

All outpatients booked for the SDCC have a surgical history and physical and a preoperative screening questionnaire $^{2}$ completed in the surgeon's office. Both are forwarded to the SDCC two weeks in advance of the anticipated day of surgery and are reviewed by the SDCC Pre-Admission nurse. The latter also has responsibility to: contact the surgeon's office as necessary to ensure completion of all sections of the history, physical and patient questionnaire, ensure that all relevant (according to SDCC guidelines) laboratory investigations have been obtained no longer than three months preoperatively (hospital policy), contact the patient to obtain additional data, and seek clarification of potential concerns. The patient is then approved for SDCC by the nurse or the patient information is referred to a staff anesthesiologist for further assessment and possible referral to the hospital Pre-Admission Clinic (PAC) for consultation. In other words, both the nurse and the second line anesthesiologist rely on the screening questionnaire and the patients chart to decide on patient suitability for outpatient surgery. Patients referred to the anesthesiologist have a Nursing/Anesthesia Consultation form completed by the nurse. This form highlights items of concern to the nurse and there is space for anesthesia orders/comments. The nurse has no specific protocol for approval of patients and uses her own clinical judgement developed after completing one year of training in the hospital PAC. Classification of patients into ASA physical status is performed by the nurse. All ASA I and some ASA II are screened by the nurse. ASA III and IV patients are referred for review of the chart and laboratory investigations by a staff anesthe-
TABLE I Details of cases that were approved by the nurse and subsequently delayed or canceled.

\begin{tabular}{llll}
\hline Age $(y r)$ & Sex & $\begin{array}{l}\text { Delay time } \\
\text { (min)/Canceled }\end{array}$ & Comments \\
\hline 54 & F & 10 & $\begin{array}{l}\text { Halothane \& multiple allergies } \\
\text { Asthma/hyperexcitable syn- } \\
\text { drome with barbiturates }\end{array}$ \\
26 & F & 5 & $\begin{array}{l}\text { Anesthetic plan altered } \\
\text { Patient ate. Given local instead } \\
\text { of general anesthesia }\end{array}$ \\
86 & M & $?$ & \\
37 & F & $?$ & Obese \& on MAOI \\
& & & Patient drank \\
67 & M & 20 & Heart murmur on examination \\
48 & M & 5 & Lawyer unaware that he was \\
27 & F & 5 & booked for spinal. \\
24 & F & 90 & Required detailed discussion. \\
33 & F & 5 & Recent stroke \\
35 & M & 5 & Severe history of PONV \\
59 & M & $?$ & Patient ate \\
& & & Drug abuse/ needle phobia/ \\
& & & HIV positive \\
23 & F & 10 & Patient ate \\
28 & F & 5 & No adult available at home. \\
36 & F & 150 & Anesthetic changed to local \\
23 & F & 10 & Patient questionnaire not com- \\
& & & pleted. On unknown \\
antidepressants
\end{tabular}

siologist working in one of the SDCC operating rooms. These patients may then be approved by the staff anesthesiologist or be referred for consultation in the PAC. The PAC is staffed by a dedicated anesthesiologist who performs a detailed history and physical examination of the patient and relevant laboratory investigations. The PAC anesthesiologist also has available other specialists for consultation as appropriate. Any patient referred to the anesthesiologist could have their ASA physical status reclassified. On the day of surgery all patients are seen by the attending anesthesiologist. After implementation of the nurse based screening model the nurse was provided with ongoing feedback and support by staff anesthesiologists. The model was evaluated one year after implementation. 
TABLE II Statistical assessment of the nurses performance compared with that by anesthesiologists with respect to whether a patient required anesthesiology assessment in the PAC.

\begin{tabular}{llll}
\hline \multicolumn{3}{c}{ Anesthesiologist view } \\
& Assessment required & Not required & Totals \\
\hline Nurses piew & & & \\
Assessment required & 34 & 83 & 117 \\
Not required & 39 & 512 & 551 \\
\hline
\end{tabular}

Sensitivity $=34 /(34+39)=46.5 \%$

Specificity $=512 /(512+83)=86.05 \%$

Positive predictive value $=34 /(34+83)=29.05 \%$

Negative predictive value $=512 /(512+39)=92.92 \%$

Positive likelihood ratio $\left(L_{R}+\right)=0.46 /(1-0.86)=3.2$

Negative likelihood ratio(LR-) $=1-(0.46 / 0.86)=0.47$

Accuracy $=(512+34) / 668=81 \%$

Prevalence $=34 /(34+39+83+512)=5.08 \%$

After institutional approval a quality audit of the model was performed prospectively in the SDCC for all patients presenting between 4th May and 29th May 1998 inclusive. For all patients, an audit form designed to evaluate the model was completed by the nurses and anesthesiologists. At the time of discharge, this audit form was attached to the Nursing/Anesthesia Consultation form if one was required for the patient. Both these forms were collected once a week and checked against the operating room schedule for SDCC. Anesthesiologists who had not completed their forms were sent a letter requesting the missing information. Forms that were missing or in which anesthesiologists failed to respond had the relevant patient charts reviewed in the medical records department. Patient charts were examined for the following information: a) whether surgery was canceled, b) whether they had been seen in the hospital PAC, c) ASA status, d) NPO status, e) surgical history and physical and preoperative screening questionnaire, and $f$ ) anesthetic and nursing record. From this information the investigators made a final decision about whether there had been any delay in the SDCC with respect to start of the case and the duration of the delay. In some cases it was not possible to estimate the exact duration of the delay due to lack of information on the audit form and lack of accurate recall on the part of the attending anesthesiologist. Rather than estimating the duration of delay, the data was coded as '?' Patients that were scheduled for local anesthesia without any anesthetic standby were excluded from the study. All canceled cases in which the study audit form was not completed were traced to determine the exact reason for cancellation. Follow up in these cases consisted of checks with the receptionists in the

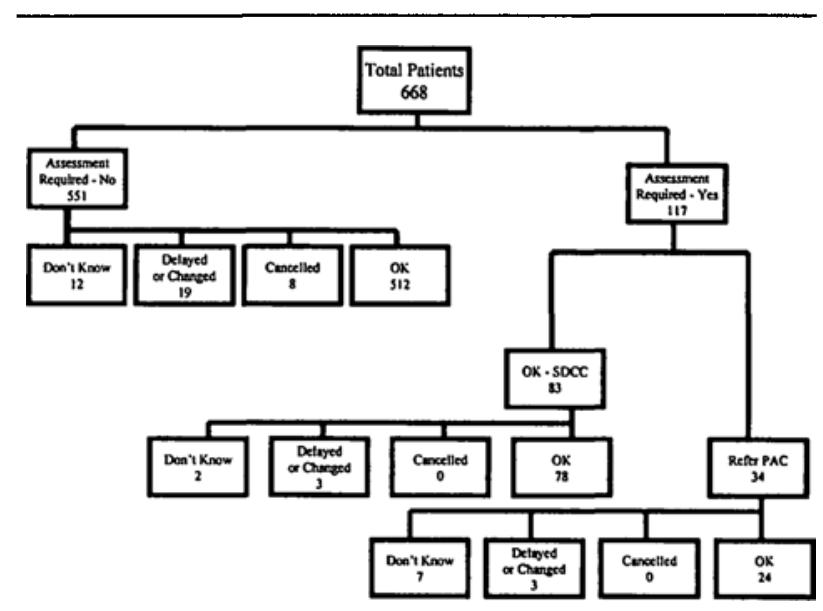

FIGURE Patient breakdown in the nurse based screening model

SDCC and examination of the medical chart. Patients who were canceled by the SDCC receptionists for not observing the 'NPO guidelines' or for not having a ride home remained in the study and were considered to be model failures. When all attempts to obtain further information were exhausted, the patients were classified as 'Don't know'.

The performance of the nurses was compared with that of the anesthesiologists using standard epidemiological methodology (Table II). ${ }^{3}$

\section{Results}

Six hundred and sixty-eight outpatients attended the SDCC during the study period (Figure). Since the hospital was closed on one week day, there were 19 days in the study period. Of these 668 patients, 551 $(82.4 \%)$ were approved by the nurse for SDCC and the remaining 117 (17.5\%) were referred to anesthesia for review. Of these $117,34(29 \%)$ were judged by anesthesia staff to require consultation in the hospital PAC, while the remaining 83 (70.9\%) were approved for SDCC and were therefore considered inappropriate referrals. Of 83 cases approved by anesthetic staff, none were canceled and three were delayed 5,10 and $10 \mathrm{~min}$ respectively for further assessment (seizures, asthma, anesthetic changed). Of the 34 cases referred to the PAC, only three were delayed for $15 \mathrm{~min}$. Reasons for delay were: patient ambivalence about surgery, patient arrived late and patient required preoperative antibiotics that had not been ordered by the anesthesiologist in the PAC. A majority, 512/551 $(92.9 \%)$, of nurse approved cases were managed in 
SDCC without any problems. Only eight of these 551 cases were canceled and another 19 were delayed (Table I). A more detailed examination of the reasons for delay revealed the following: five patients ate/drank and the rest had problems that could have been identified with a more thorough work up. Two important reasons for canceling cases were: eating (3) and social reasons $(2)$.

\section{Discussion}

We have demonstrated that a nurse based model for screening all outpatients in a large university affiliated tertiary hospital performed with an accuracy of $81 \%$, a specificity of $86.05 \%$ and a negative predictive value of $92.92 \%$. The cancellation rate was $1.4 \%(8 / 551)$ and the case delay rate was $3.4 \%(19 / 551)$. The referral rate to anesthesiology staff was $17.5 \%(117 / 668)$ and the referral rate to the PAC was $5.08 \%(34 / 668)$. The model was better at 'ruling out' patients who did not need to be seen by anesthesiology ahead of the day of surgery rather than 'ruling in' patients who needed to be seen.

The questionnaire utilized was very similar to that described by Badner. ${ }^{2}$ We did not assess the effectiveness of this questionnaire because it has been shown to be highly sensitive and specific. ${ }^{2}$ The study was designed to assess the effectiveness of our nurse based model. Before we implemented this model, all SDCC patient histories were reviewed by anesthesiologists and consultations were performed in the PAC as indicated or when requested by the surgeon. However, the cancellation and delay rates are not available because there was no formal evaluation of the anesthesiology based approach. A 5\% delay rate and a 3\% cancellation rate have been reported from pre-admission clinics run by anesthesiologists ${ }^{4}$ where patients were referred by surgeons and were not limited to ambulatory patients. Delay rates as high as $8.6 \%$ have been reported in other pre-admission clinics. ${ }^{1}$ We did not collect data on patient age, ASA status or surgical procedure, since, it has been found that ASA class and surgical intensity do not allow accurate determination of the need for pre-anesthesia interventions in outpatients. ${ }^{5,6}$ Comparisons with other models should also be made with caution as the time period and populations may differ.

Anecdotal opinion among anesthesiologists would suggest that nurses cannot perform preoperative evaluations as well as physicians. ${ }^{7}$ Our results suggest that such opinions may not be valid. The low positive predictive value may be due in part to our requirement that the nurse refer all ECGs for interpretation to the staff anesthesiologist and because of our difficulty in provid- ing uniform guidelines that would help the nurse overcome ambiguity consequent to discretionary physician management. Recently it has been shown that there is little agreement among anesthesiologists with respect to clinical decision making. ${ }^{8}$ The requirement to see a patients's ECG in advance was assessed by means of a questionnaire in our department and a majority $(80 \%)$ of the anesthesiologists still expressed a preference to see these ECGs. A common theme prevalent in the responses was that the ECG was being used as a surrogate measure of fitness in order to compensate for a lack of adequate history and physical from the surgeon. This is troubling because a normal ECG does not guarantee absence of underlying heart disease and because the ECG has not been evaluated for such triage. In addition, it has been demonstrated that there is little agreement among anesthesiologists with respect to the need for an ECG. ${ }^{8}$ It is suggested that formulation of evidence based clinical algorithms may help reduce unnecessary referral to anesthesiologists by our model. The nurse based model also has the advantage of providing a consistent approach to significant preoperative issues and adhering to them in dealing with patients, surgeons and third parties. Deviations from such a consistent approach would occur when multiple anesthesiologists are involved in discretionary decision making and would undermine the confidence of all parties in the anesthesia department. This was an important problem with our traditional anesthesiology based approach.

At present our nurse does not have a role in ordering relevant tests or canceling unnecessary laboratory tests. Previous studies ${ }^{9}$ have shown that a nurse practitioner, given appropriate guidelines, was able to cancel 338 unnecessary tests of 1,606 total tests ordered by surgeons in 1,503 PAC patients and thereby realize cost savings of $C \$ 5,376$. The potential for canceling unnecessary tests in our SDCC is limited because most tests have already been completed when the patient is booked for the SDCC. In addition, widespread publication of provincial guidelines have helped to reduce unnecessary preoperative testing in surgical patients. ${ }^{10}$ Expansion of the nurses' role would therefore be limited to ordering tests according to specific guidelines. The prevalence rate for anesthesia consults as identified by the nurse practitioner in our study of $5.08 \%$ compares favourably with a rate of $2.9 \%$ reported with a same day admit/discharge program in another Canadian teaching hospital. ${ }^{9}$ In addition, the referral rate to anesthesiology staff of $17.5 \%$ approximates the prevalence rate of ASA III plus IV patients in our SDCC. This suggests that our nurse based model would perform similarly in other units with comparable prevalence rates and patient demographics. 
Other potential benefits of the nurse based model include: a) providing preoperative teaching, b) identifying problems which could affect recovery/discharge, c) ensuring complete pre-admission documentation, d) ensuring compliance with preoperative medication requirements, e) allowing early discharge planning, f) decreasing inpatient admissions by appropriate identification and implementation of patient specific resource planning, g) minimizing OR delays and cancellations by early identification of problems, $h$ ) initiating early care planning for patients with special needs, i) providing guidance for sequencing of the OR schedule so that system resources are better matched with demands, $j$ ) allowing early referral to third parties (example social workers, home care nurses) and, $k$ ) ensuring that old charts of complex patients are available for anesthesiology review.

Currently, our SDCC processes 30-60 patients a day. Under the traditional model, all of these histories would have to be reviewed by anesthesiology staff. With the nurse based model, most of the histories are reviewed by the nurse and only $17.5 \%$ are referred to anesthesiology. Examination of the reasons for delays and cancellations (Table I) reveals that the nurse needs to be more vigilant in reinforcing the fasting guidelines, ensuring adequate social support and in obtaining a complete history on telephone contact.

An important limitation of the study is that we did not examine factors such as unanticipated intra-operative events (UIE) with the nurse based model. However, the incidence of such events is very low (4.14\%) after PAC assessments and it may not be a worthwhile exercise in outpatients because outpatients have a lower risk of UIEs compared to PAC patients. ${ }^{11}$ Blinding was not possible in the study because it would have removed important information upon which decisions are made and would not be reflective of the model's performance.

In conclusion, a nurse based model represents an effective way of screening surgical outpatients. The model's performance was found to be satisfactory in our surgical day care centre. The model has the potential to streamline preoperative anesthetic assessment and allow for more efficient use of anesthesiologist's time. In addition, it has the potential to improve the overall care of outpatients and the efficiency of outpatient facilities.

\section{Acknowledgments}

We wish to thank the members of the Department of Anesthesia for their assistance and Marsha Radvak for implementing the nurse based model in our surgical day care centre.

\section{References}

1 Pasternak LR. Screening patients: strategies and studies. In: McGoldrick KE (Ed.). Ambulatory Anesthesiology - A Problem Oriented Approach, 1st ed. Baltimore: Williams \& Wilkins Inc., 1995: 2-19.

2 Badner NH, Craen RA, Paul TL, Doyle JA. Anaesthesia preadmission assessment: a new approach through use of a screening questionnaire. Can J Anaesth 1998; 45: 87-92.

3 Schechter $M T$, Sheps SB. Diagnostic testing revisited: pathways through uncertainty. CMAJ 1985; 132 : 755-60.

4 Conway JB, Goldberg J, Chung F. Preadmission anaesthesia consultation clinic. Can J Anaesth 1992; 39: 1051-7.

5 Davis JE, Sugioka $K$. Selecting the patient for major ambulatory surgery. Surgical and anesthesiology evaluations. Surg Clin North Am 1987; 67: 721-32.

6 Mattison RA, Fleck C. Predicting the timing of ambulatory preanesthesia evaluation by ASA classification and surgical intensity score. Anesthesiology 1997; 87: A24.

7 Miller $R D$, Rampil $L$, Cohen N. Fewer residents: financial, educational, and practical implications. Anesth Analg 1998; 87: 242-4.

8 Down MP, Wong DT, McGuire GP. The anaesthesia consult clinic: does it matter which anaestherist sees the patient? Can J Anaesth 1998; 45: 802-8.

9 Hurtado C, Chung F, Krishnathas A. Does the nurse practitioner have a role in the pre-admission unit? Can J Anaesth 1996: 43: A61B.

10 Protocol for Electrocardiograms. Protocol Steering Committee. PSC 9612-004p; April 1st; Medical Services Commission and British Columbia Medical Association. 1997.

11 Lee A, Lum ME, Perry M, Beeban SJ, Hillman KM, Bauman $A$. Risk of unanticipated intraoperative events in patients assessed at a preanaesthetic clinic. Can J Anaesth 1997; 44: 946-54. 